Digilec 8 (2021), pp. 21-35

Fecha de recepción: 11/06/2021

Fecha de aceptación: 28/05/2021

DOI: https://doi.org/10.17979/digilec.2021.8.0.8568
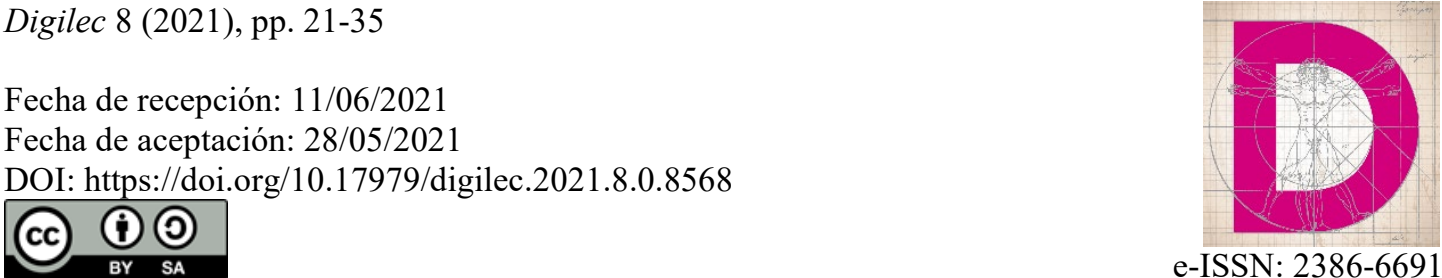

\title{
(RE)PENSANDO LAS POLÍTICAS LECTORAS, PROGRAMAS DE FOMENTO Y MEDIACIÓN DE LA LECTURA DESDE LA JUSTICIA SOCIAL
}

\author{
RE-THINKING READING POLICIES, PROGRAMS TO PROMOTE \\ AND MEDIATE READING FROM PERSPECTIVE OF SOCIAL \\ JUSTICE
}

Concepción LÓPEZ-ANDRADA*

Centro de Estudios Latinoamericanos de Educación Inclusiva (CELEI, Chile)

Universidad de Extremadura (España)

Orcid: http://orcid.org/0000-0002-9423-0434

\section{Aldo OCAMPO-GONZÁLEZ**}

Centro de Estudios Latinoamericanos de Educación Inclusiva (CELEI, Chile)

Orcid: https://orcid.org/0000-0002-6654-8269

\begin{abstract}
Resumen
El artículo analiza algunas de las tensiones teóricas y epistémicas que se encuentran en el interior de las políticas lectoras, programas de fomento y mediación de la lectura del presente desde la óptica de la justicia social. Emerge en este trabajo la necesidad de romper con la gramática escolar hegemónica, en particular, con el énfasis de asimilación imperceptible con la cultura dominante de ciertos colectivos comprendidos como no culturalizados o no competentes en materia de lectura. Atravesando estas problemáticas se hace necesario crear nuevos formatos lectores -concebidos en términos de transformación-, que permitan recuperar la construcción de ciudadanía y justicia a través de las prácticas de lectura en su definición más extensa y plural. La lectura constituye un objeto crítico clave en la lucha por la ampliación de los campos de desarrollo democrático, con relación a la emergencia de un nuevo terreno que permita la cristalización de una praxis dialógica que asuma la voz de la diferencia y la multiplicidad.
\end{abstract}

Palabras clave: Lectura; alfabetización; fomento de la lectura; inclusión; diferencia; justicia social.

\footnotetext{
* Facultad Formación del Profesorado. Universidad de Extremadura. Avd. de la Universidad, s/n. 10003 Cáceres. Email: clopezc@unex.es concepcion.lopez.andrada@celei.cl

** Carmen 566, Of. 1907, Santiago de Chile, Chile. Email: aldo.ocampo@celei.cl
} 


\begin{abstract}
The article analyses some of the theoretical and epistemic tensions of reading policies, the programs to promote and mediate reading nowadays from the perspective of social justice. In this study the need to break with the hegemonic school grammar emerges, in particular, with the emphasis of the imperceptible assimilation with the dominant culture of certain groups, as they are understood as not culturalized or not competent in reading. After assessing these problems, it is necessary to create new reading formats -conceived in terms of transformation - that allow the recovery of the construction of citizenship and justice through reading practices in their broadest and most plural definition. Reading constitutes a key critical object in the struggle for the expansion of the fields of democratic development, which is in relation to the emergence of a new area that allows the crystallization of a dialogic praxis assuming the voice of difference and multiplicity.
\end{abstract}

Keywords: Reading; literacy; reading promotion; inclusion; difference; social justice. 


\section{INTRODUCCIÓN: LECTURA Y ECOLOGÍA DE SABERES}

Habitamos un presente caracterizado por los discursos mediatizados y fragmentados, por esa modernidad líquida (Bauman, 2007) que impregna nuestras relaciones con los otros; una sociedad del riesgo ${ }^{1}$ que se singularizada por la incertidumbre y por los procesos de crisis, donde la lectura se revela como un mecanismo intelectual que expande el ejercicio de la ciudadanía dentro de las posibilidades emancipadoras de la consciencia crítica (hooks, 2017), asociada a la proliferación de nuevas formas de expresiones ciudadanas -específicamente de naturaleza micropolítica y performativa- que articulan mecanismos de agenciamientos alternativos ${ }^{2}$.

El estudio de la lectura en tanto práctica cultural, educativa y social interpela una ecología de saberes, es decir, siguiendo a Sousa (2010), la articulación de sistemas intelectuales que afiancen alternativas viables para redistribuir el derecho a la educación y a la lectura desde la justicia social, conforme a lo que cada persona y grupo social requiere. La ecología de saberes visibiliza los medios de interacción entre diversos saberes para confluir en el ensamblaje analítico-metodológico de esta propuesta. Vinculamos el desarrollo de este articulo a la noción de pensamiento alternativo de las alternativas en materia de derecho a la lectura, en tanto, conocimiento-comointervención-en-la-realidad (Sousa, 2010), cuya gramática obliga a ubicar su centro en torno a la dimensión ético-política en cuanto a la estructuración de un proyecto de educación lectora orientado a deconstruir la visión hegemónica de la cultura letrada que atraviesa la mayoría de los programas de formación a nivel global.

Pensar en torno al papel de las intervenciones en el mundo a través de la lectura requiere de otras formas de saberes invisibilizados, devaluados o excluidos de las estructuras educativas. La construcción de una agenda investigativa, política y epistemológica en torno al pensamiento alternativo de las alternativas en materia de educación lectora, ciudadanía, acción política y redistribución enfrenta como primer obstáculo la identificación de saberes que posibilitan o bien, restringen, la articulación de una intervención crítica en la vida de cada lectora o lector, en sus agencias, procesos de biografización y trayectorias, así como, en la escuela y en la sociedad (Ocampo-González y López-Andrada, 2020).

Este trabajo tiene como objetivo el análisis de las tensiones que se encuentran en el interior de las políticas lectoras y planes de fomento de la lectura en donde la hibridación de prácticas -orales y escritas, de lectura y de escritura-, y de formatos está ocupando un espacio propio de cohabitación en el presente. El desarrollo de la lectura como práctica relacional se articula en escenarios que van más allá de la institución educativa. Espacios

\footnotetext{
${ }^{1}$ El sujeto contemporáneo sufre un proceso de individualización en el cual se desvincula de formas tradicionales comunitarias (Beck, 1998).

${ }^{2}$ Este trabajo se adscribe a la noción de alternativa proporcionada por Sousa (2010). Para este autor es necesario forjar un pensamiento alternativo sobre las alternativas, evitando reproducir implícitamente un conjunto de valores y contenidos incapaces de intervenir la realidad desde otros sistemas de razonamientoReferimos en esta oportunidad a un conjunto de alternativas contrahegemónicas.
} 
en ocasiones novedosos, ligados al entretenimiento y al ocio, pero también vinculados a la creación de redes, comunidades y espacios de sociabilidad.

Otro elemento clave entre las dinámicas del presente será el acceso a la información. En un mundo digital que construye y deconstruye subjetividades, genera nuevos controles, seduce al poder y se torna cómplice absoluto de la vigilancia (Araújo \& Cardozo, 2016, p. 210) se están resignificando ciertos vínculos comunitarios y redes de socialización. Una paradoja se ocasiona respecto a los nuevos modos de convivencia social en donde se remarcan las diferencias en un mundo cada vez más homogéneo en el que a partir de las tecnologías se modela la idea de eficiencia, acceso, planificación y racionalidad.

La denominada brecha digital se ha convertido en un concepto ligado a los más recientes trabajos sobre introducción de las tecnologías de la información en la sociedad y especialmente en el sistema educativo a través de la idea de que estos dispositivos deben llegar a toda la ciudadanía para superar las desigualdades implícitas. Una reformulación de la brecha digital resultará indispensable en un presente caracterizado por la traslación de las dinámicas laborales precarias a las políticas y programas educativos en sintonía con ese sujeto del rendimiento que se autoexplota (Han, 2017).

El acceso a la información se ha convertido en un componente clave y a la vez una manera de medir las desigualdades en la sociedad actual. La brecha digital es un reflejo de los conflictos sociales, por ello, la brecha digital alberga también una brecha social. Una brecha digital social que alude a contextos económicos, políticos, sociales y culturales concretos y singulares que deben ser tenidos en cuenta a la hora de estudiar su propia dimensionalidad, pero cuya respuesta a través de los indicadores estadísticos estandarizados de los Organismos Internacionales tiende a la homogenización. Autores como Hirtt (2010) ha propuesto una revisión crítica de las políticas educativas en el ámbito mundial, arrojando un análisis sobre el enfoque de competencias promovido por organismos internacionales como la $\mathrm{OCDE}^{3}$, lo que conlleva una interpretación de la educación como factor al servicio de la competencia económica cuyo propósito principal consiste en el establecimiento de dispositivos de certificación que se adapten a las necesidades de desarrollo de competencias y a la supuesta flexibilidad del mercado laborar (Hirtt, 2010, p. 113).

En el capitalismo cognitivo se establecen relaciones de desigualdad y brechas sociales que se generan en su estructura misma en un orden al que no únicamente se fuerza a la gente, a los cuerpos y a las cosas, sino en el que, además, estos juegan simultáneamente un papel activo (Lorey 2008, p. 63-64); por lo tanto, el estudiante y/o

\footnotetext{
${ }^{3}$ La OCDE es el organismo que unifica, homogeniza y cuantifica las políticas educativas estableciendo una agenda internacional a través de informes descriptivos, comparaciones, evaluaciones y recomendaciones. Los trabajos críticos de Rizvi \& Lingard, 2009a; y Rizvi \& Lingard, 2009b analizan las políticas educativas ubicadas dentro del nuevo terreno de la globalización. Estas políticas han sido profundamente afectadas por las transformaciones sociales y económicas acontecidas en las dos últimas décadas, y según los autores, los gobiernos nacionales han tratado de ajustar sus prioridades educativas a los imperativos de la globalización. De este modo, estas investigaciones exploran los controladores claves del cambio en las políticas educativas y sostienen que estos no funcionan de la misma manera en todos los Estados; asimismo, los autores identifican las políticas educativas vinculadas a las políticas neoliberales y hacen una descripción de lo que llaman "el comercio mundial de la educación".
} 
trabajador se ajusta a un mundo competitivo mediado por el capital en el que "las ideas de autonomía y libertad están constitutivamente conectadas con los modos hegemónicos de subjetivación en las sociedades capitalistas occidentales" (Lorey 2008, p. 58). Al atravesar la categorización del sujeto como lector/consumidor de contenidos digitales y del usuario acrítico de herramientas tecnológicas será ineludible trascender la lógica instrumental.

\section{LA CRISIS DE LA CRÍTICA Y LA LECTURA INSTRUMENTAL: EL PARADIGMA PISA}

Vivimos en sociedades en crisis y en sociedades caracterizadas por la crisis de la crítica. La filósofa catalana Marina Garcés expone que somos analfabetos ilustrados: tenemos a un clic todos los conocimientos de la historia de la humanidad, pero vivimos incapacitados para la crítica y la acción (Garcés, 2017). En las situaciones de crisis, los discursos enfatizan las diferencias teóricas y los antagonismos políticos (Chartier, 2004); los productos culturales se han ido transformando en ese bien mercantil destinado a satisfacer los gustos de los clientes.

El modelo educativo imperante en la actualidad se caracteriza por la proyección de programas de alfabetización vinculados a las demandas económicas, es decir, un modelo educativo orientado al mercado de trabajo, reducido al aprendizaje de habilidades. Así pues, las formas en las que se produce y se reproduce el conocimiento vienen determinadas por relaciones de poder más amplias, que sirven a una lógica instrumental, a la racionalidad alienante de la línea de ensamblaje (Giroux, 1992, p. 259). Un sistema educativo bajo demanda de la eficiencia y de la cuantificación de la práctica escolar que esconde un proceso burocrático (Liria, Fernández \& Ferrández, 2017). De modo que nos encontramos ante una institución educativa en crisis, y en la que los discursos de la innovación han tapado realidades educativas conflictivas y en permanente disputa.

Las encuestas e informes que regularmente aparecen sobre consumo de libros, hábitos lectores, comprensión y competencia lectora indican un futuro incierto que de manera general tiende a la culpabilización de sujetos e instituciones. Al abordar la construcción de representaciones es interesante tener en cuenta elementos relacionados con la recepción y la circulación de resultados internacionales sobre competencia y comprensión lectora, cuyo modelo paradigmático es el Informe PISA ${ }^{4}$, y su tratamiento en los medios. El criterio que determina la difusión de estos resultados, los verdaderos beneficiados de estas evaluaciones internacionales externas o el efecto que suscita en las representaciones de la lectura y con relación a los lectores en situación de aprendizaje resulta, en ocasiones, borroso e impreciso; asimismo, serán preguntas pertinentes de cómo

\footnotetext{
${ }^{4}$ El test PISA (Programme for International Student Assessment) consiste en una prueba trianual a alumnos de 15 años (en la mayoría de los países participantes próximos a la finalización de la escolarización obligatoria) establecida por la OCDE (Organización para la Cooperación y el Desarrollo Económicos). Tiene como objetivo principal generar indicadores sobre principios del rendimiento educativo adoptando una perspectiva "competencial" que con relación al desarrollo de habilidades lectoras se focaliza en la interpretación y comprensión de diversos materiales escritos que serán usados por el alumnado en su vida diaria (Turner, 2006).
} 
la articulación de significados excluye, en ocasiones, a los propios protagonistas de estas prácticas lectoras (Bombini, 2008, p. 26).

La práctica educativa toma su forma como variable económica, como motor para el desarrollo y el crecimiento económico. Se produce, pues, una subordinación a elementos de la mundialización económica y de la valorización capitalista manifestados en la competición por puntuar lo más alto posible en los rankings a través de la mejora de la calidad y excelencia educativa, terminología utilizada en este tipo de retórica vaciada de significado $^{5}$. PISA acentúa el aprendizaje al margen del currículo escolar (FernándezCano, 2016). Lo que en términos de evaluación imposibilita la capacidad de converger estos resultados al ámbito de las micropolíticas educativas de cada país (Ball. 1989; Prais, 2003). Por otro lado, las limitaciones metodológicas de PISA se extienden a la opacidad de la muestra, a la incorrecta enunciación de las hipótesis correlaciónales y a la cuestionable validez de los instrumentos de medida (Fernández-Cano, 2016). La variante para adultos (16 a 65 años) de las pruebas PISA es el Programme for the International Assessment of Adult Competencies (PIAAC), iniciativa de la OCDE que consiste en una evaluación internacional y estandarizada con relación a la comprensión lectora ${ }^{6}$ y los componentes de lectura, a la capacidad de cálculo y a la resolución de problemas en entornos digitales.

Desde esta perspectiva, una de las tensiones más significativas que atraviesa las evaluaciones externas y estandarizadas consiste en negar la singularidad que define cualitativamente el papel de las prácticas lectoras de cada estudiante. En este sentido, se observa la coexistencia de un dispositivo de homogenización sobre los aspectos sociocognitivos implícitos en el acto de leer. En este contexto, las evaluaciones de la comprensión lectora -capacidad para decodificar un texto y captar la intención- y de la competencia lectora -capacidad para utilizar y aplicar la información en diversos ámbitos-, desconocen el papel del desempeño sociocognitivo que cada lector posee en relación con el acto de lectura.

Las dificultades de acceso y manipulación de la lectura tienden a regenerar el ideal esencialista para explicitar sus conflictos, cuyas acciones discursivas esconden las contradicciones de la estructura escolar, atribuyendo que son problemáticas individuales propias de cada estudiante. En palabras de Young (2002), estas discordancias pueden explicarse mediante una creencia que resalta la naturaleza de lo diferente, atribuyendo de esta forma, un estatus de inferioridad a quienes no logran los estándares esperados. Para Young (2002) las políticas de la OCDE en relación a los sistemas de medición de la competencia lectora perseveran en la reducción del capital cultural diversificado a una

\footnotetext{
${ }^{5}$ En el contexto del estado español, la reciente y controvertida Ley Orgánica para la Mejora de la Calidad Educativa (LOMCE) funciona como paradigma de esta legitimación de discursos a través de las premisas impuestas por la OCDE. La "ideología de la privatización", es decir, la apertura del sector público a la intervención de agentes privados (Fernández-González, 2015), se añade a otras características de estos procesos de políticas globales como son la estandarización del rendimiento educativo y la implantación de evaluaciones externas (Casanova y Navas, 2015).

${ }^{6}$ Cabe señalar que la concepción de la "comprensión lectora" en estos informes auspiciados por la OCDE no difiere del concepto de "competencia lectora" o de "capacidad lectora", que en su versión original en inglés se define como: "Reading literacy is understanding, using, and reflecting on written texts, in order to achieve one's goals, to develop one's knowledge and potential, and to participate in society" (OECD, 2009, p. 23).
} 
media de lo común, estableciendo una estrategia que construye como poco competentes -asignando la representación de Kaplan (2012) referida a buenos y malos estudiantes- a aquellas comunidades y estudiantes que están por debajo de los indicadores y las puntuaciones legitimadas sociopolíticamente por cada organismo externo.

Es tarea crítica de las administraciones, centros educativos, docentes e investigadores la creación de herramientas para desarticular las clasificaciones y expectativas que se esconden en las interpretaciones de los resultados escolares, adquiriendo la forma de esquemas clasificatorios; permitiendo y simplificando sus medios de distinción y categorización. Se observa que la capacidad para organizar en categorías los datos posee una importante función económica (Kaplan, 1992). De igual modo, los sistemas de evaluación estandarizadas al medir a todas las personas con criterios universales, articula una concepción dicotómica de tipo jerárquico en relación con la diferencia, el rendimiento escolar, la cognición humana, la labor de los docentes y la legitimidad de las propias instituciones educativas. Todas estas manifestaciones no son otra cosa que una expresión de las ideologías objetivistas de la justicia social y educativa (Young, 2002). En ella, los devaluados son aquellos que obtienen bajos resultados, o bien son oprimidos por pertenecer a sectores con menos oportunidades culturales, evaluadas arbitrariamente por los marcos de valores hegemónicos de la cultura dominante. Nos preguntamos, entonces: ¿qué es aquello que es legítimo de ser considerado una experiencia cultural válida?

En esta posición, los grupos privilegiados a juicio de Young (2002) se tornan neutros, mientras que los marginalizados y subalternos basan su "existencia" en un conjunto reducido de posibilidades. Cada comunidad escolar, inscribe su tarea educativa y formativa en condiciones histórico-culturales particulares. Comúnmente, los resultados de estas evaluaciones externas son empleados como círculos condenatorios de las comunidades educativas, incrementando la devaluación sociocultural al no lograr ingresar en determinadas clasificaciones.

Con relación a la construcción de una ciudadanía lectora en el ámbito de las políticas públicas educativas será fundamental remarcar que las evaluaciones sobre competencia lectora deberán adscribirse a una afirmación positiva de la diferencia, convirtiéndose así, en un eje transversal de los programas y planes de educación lectora. Desde otra óptica, las evaluaciones internacionales inscritas en una gramática de homogenización han de repensarse sobre aspectos relacionados con: (1) los sistemas de diversificación de las condiciones de exclusión, opresión e injusticia; (2) la creación de estrategias que permitan reconocer cómo hacer frente a cada una de ellas, a través de los resultados obtenidos y (3) la construcción de indicadores de calidad de la enseñanza y de la tarea educativa inspirada en una ontología pluralista (Ocampo-González, 2016).

En este apartado se ha expuesto como PISA considera la competencia lectora como impulsora del desarrollo económico (OECD, 2009), que examinada junto a la idea freiriana de la "lectura del mundo" se identificaría sin ninguna duda con ese carácter instrumental de la lectura. En este punto cabe cuestionarse si el paradigma de la OCDE, tendencia hegemónica en el desarrollo de las políticas educativas en la que prima lo instrumental, la herramienta como punto central de la innovación tecnológica, la obsesión 
por la eficiencia de la pedagogía por objetivos ha penetrado no solo en los discursos, sino también en las prácticas de lectura.

\section{CONFIGURACIÓN DE UNA POLÍTICA DE EDUCACIÓN LECTORA Y DE DERECHO A LA LECTURA DESDE LA JUSTICIA SOCIAL}

Las intervenciones que emergen del pensamiento alternativo de las alternativas, han de reforzar sus herramientas con el objeto de dislocar los modos dominantes de aproximación y ejercicio de la cultura letrada hegemónica, traducidos en modos particulares de disposición de los sistemas de alfabetización inicial, de ejercitación de la comprensión y de la competencia lectora, de la educación literaria y del canon como eje transversal de continuidad de los marcos de valores que sustentan la principal tarea de la cultura letrada dominante.

Una intervención concreta y subversiva requiere de saberes alternativos que, debido al carácter emergente del campo aquí descrito, enfrentan la compleja y contingente tarea de construirse. Una política de educación lectora coherente con estos planteamientos asumirá que "la preferencia debe ser dada a la forma de conocimiento que garantice el mayor nivel de participación a los grupos sociales involucrados en su diseño, ejecución y control, y en los beneficios de la intervención" (Sousa, 2010, p. 56).

En cierta medida, sostenemos que los marcos disciplinarios y discursos que confluyen en la organización del campo de estudio de la lectura tienden a reproducir mecanismos de esencialización a través de sus planteamientos, incurriendo en ocasiones, en reduccionismos y en una división simbólica del mundo. El campo de estudio del derecho a la lectura carece de inscripciones epistémicas y metodológicas inteligibles que permitan situar sus tareas frente a la multiplicidad de diferencias a las que intenta legitimar. En este marco de interpretación, la lectura se convierte en un fenómeno estructural. Esto es, un problema de funcionamiento social, no así, como un problema técnico, basado en políticas de absorción de colectivos minoritarios que, a través de una inclusión ficticia orientada a incluir a un conjunto de estructuras de escolarización y culturización que imperceptiblemente, los obliga a adoptar los marcos de valores legitimados por la cultura dominante. En definitiva, concebir la lectura como un fenómeno estructural sugiere crear dispositivos metodológicos para comprender la naturaleza y el funcionamiento de la exclusión, la opresión, la dominación, en tanto, dimensiones comunes y transversales de la experiencia social y escolar. La lectura concebida en términos de fenómeno estructural sugiere atender al conjunto de prácticas a través de las cuales determinados grupos son excluidos de este derecho. Un análisis con estas características inquiere en el develamiento de la función ideológica -en el contexto hegemónico y contrahegemónico-, la emergencia de nuevas formas de dominación que pueden obliterar a través de su dimensión distributiva y las estrategias requeridas para organizar un proceso de emancipación a través de la política de la diferencia. Consecuentemente, será también necesario examinar estas tensiones en referencia a las racionalidades que aportan los nuevos movimientos sociales.

Las políticas educativas (Taylos, Henry, Lingard y Rizvi, 1997; Rizvi y Lingar, 2009), sociales y culturales han adoptado un enfoque del derecho aplicado a los bienes 
culturales desde una perspectiva de valor colectivo, enfatizando en una totalidad homogeneizadora que concibe las diferencias como eje de diferenciación social agudizando las condiciones de opresión, dominación e injusticia-, implantando, pues, una concepción del derecho a la lectura en bloque cuya racionalidad restringe el potencial de la redistribución y la diferencia. En tal caso, será un elemento clave para la movilización considerar los programas y mediaciones lectoras desde la potencia emancipatoria que otorga herramientas metodológicas para identificar las condiciones de opresión -trabas en el autodesarrollo- y de dominación-trabas en la autodeterminación- (Young, 2002). Consecuentemente, el carácter sistémico y multidimensional que atraviesa la comprensión de la lectura como fenómeno estructural, sugiere atender a la red de relaciones entre grupos, a la emergencia de una multiplicidad de capitales culturales diseminados en la trama societal. Cada institución educativa forja un determinado capital cultural y un destino social.

Parte de la tarea crítica que enfrenta el derecho a la lectura, consistirá en hacer emerger sistemas intelectuales que permitan comprender cómo operan los procesos institucionales sistemáticos que impiden a determinados colectivos disfrutar de los bienes culturales, al tiempo que dichas tecnologías de relegamientos limitan sus capacidades y posibilidades de acción, incidiendo en el destino social de la multiplicidad de colectivos y grupos sociales y en la creación de nuevos horizontes políticos y culturales que permitan albergar en la trama societal algunas de las ideas esbozadas en este trabajo. Todo ello exige una descripción del corpus de tecnologías que participan en la proliferación de procesos de marginación de modos diferentes de lectura en la experiencia escolar.

¿Qué supone pensar relacionalmente el derecho a la lectura?, para responder a esta cuestión recurrimos a la contribución de la corriente interseccional ampliamente difundida por autoras tales como Hill Collins (1990), Brah (2014) o Yuval-Davis (2014). La construcción de la categoría analítica del derecho a la lectura requiere metodológicamente de la articulación de un modelo de análisis categorial no opresivo que respete las diferencias. Será necesario crear categorías políticas específicas y contingentes que ayuden a pensar relacionalmente; con el objeto de subvertir las lógicas colonialistas, imperialistas y capitalistas que alojan en su centro de actividad la noción de justicia educativa y social e inclusión, contribuyendo a la imposición de categorías ahistóricas y esencialistas para emprender la búsqueda del sujeto al interior de la justicia y la inclusión.

Esta visión de la justicia cultural y la ciudadanía lectora explicita la necesidad de descentralización sistemática del sujeto normativo (Spivak, 2008) que estructura las agendas de investigación, al tiempo que logren dar cuenta cómo las políticas del libro (Fraatz, 1987; Perfetti, 1991; Edmondson \& Shannon, 2002), los programas de animación a la lectura y en especial, las agendas de investigación tienden a omitir la experiencia de las múltiples diferencias o grupos sociales significados como diferentes, imponiendo una lógica denominada como esencialismo liberal. En particular, cómo las maneras en que la experiencia de ciertos lectores es marginada a través de los programas de fomento y mediación de la lectura. Cada una de estas relaciones es atravesada por la interseccionalidad. Un programa de animación a la lectura debe explorar los dispositivos de micro praxis que generan tecnologías de cooptación de determinados colectivos desplazándolos a las fronteras del derecho a la lectura. Es necesario de igual manera, 
ofrecer una exploración en torno a los macro y micro- regímenes del poder y de los diversos ejes de diferenciación que son instituidos en formaciones estructurales específicas, ámbitos expresos en la determinación de oportunidades y de nuevas formas de legitimidad y de legibilidad social. Por ellos, nos preguntamos: ¿cómo ampliar oportunidades a través de la lectura?, intentando responder a esta interrogante, suscribimos en este trabajo, a la noción de diferencia propuesta por la corriente derridiana, enfatizando en la concepción de proceso y devenir permanente.

Las políticas de fomento de la lectura desde una perspectiva de equidad cultural e inclusión han reafirmado un interés asimilacionista a grupos que históricamente han quedado fuera de los cánones literarios y lectores proporcionados por la cultura dominante, intentando absorberlos a intereses que rechazan o supeditan sus medios de aproximación a la comprensión del mundo. Tales políticas del libro y del fomento de la lectura se inspiran en gramáticas que apelan por el progreso, al tiempo que permiten nuevas formas de homogenización y de devaluación de colectivos de lectores otros.

\section{EL PENSAMIENTO RELACIONAL PARA EL DISEÑO DE POLÍTICAS DE LA LECTURA Y PROGRAMAS DE ANIMACIÓN A LA LECTURA}

Los argumentos expuestos en los apartados anteriores permiten reconocer que la lectura como praxis social y cultural, devine en la focalización y comprensión de los impedimentos sistemáticos que afectan relacionalmente al ejercicio de este derecho. Para Young (2002), los derechos constituyen bienes no-materiales, como tal, asumirán la pregunta: ¿qué significa redistribuir un derecho? Para comprender el rol del derecho aplicado a los bienes culturales, las artes y la información, será necesario analizar la naturaleza de la redistribución, pues, esta se alinea con una política basada en el pluralismo y la multiplicidad. Tal como señala Lazzarato (2006) la política occidental se fundó sobre una lógica homogeneizadora que enfatiza en el valor colectivo, es decir, en la totalidad y la universalidad.

El pensamiento relacional es clave en el diseño de políticas de lectura y programas de animación a la lectura, puesto que, permite examinar cómo operan las estructuras de culturización y socialización - de carácter dinámicas y en permanente movimiento- y sus dispositivos de arrastre a los márgenes del derecho a la lectura. La tarea crítica que enfrenta la praxis desde la lectura desde una perspectiva social y política, consiste en el develamiento de nuevas formas intelectuales y metodológicas que interroguen los modos de pensar, experimentar y practicar la educación lectora desde la cristalización de nuevas espacialidades educativas y estrategias que contribuyan a movilizar nuevas racionalidades en la problematización de la alfabetización y la escolarización, el desarrollo ciudadano y político, así como, interrogar acerca de las posibilidades que proporcionan los marcos y vocabularios existentes ante la heterotopicalidad de tensiones analítico-metodológicas que atraviesan su campo de producción.

Una política del libro, de educación lectora y de fomento de la lectura ha de incurrir en la creación de herramientas analíticas para discurrir y disputar el legado de la cultura dominante al referir a la praxis lectora. En este sentido, recurrimos a la necesidad de superar el problema técnico de la inclusión y de la lectura, caracterizado como una 
modalidad altamente esencialista y en cierto modo, contrarias a las demandas de una inclusión real. La aplicación del análisis interseccional (Anthias, 1983) al examen de las condiciones que restringen el ejercicio de los derechos educativos, culturales y de la lectura, no sólo beneficia a la población que se encuentra en sus márgenes, sino más bien, da pistas para examinar cómo la totalidad concebida como singularidades múltiples es afectada por diversas expresiones -regenerativas y performativas- del poder. Se convierte así, en un eje de tematización clave en el estudio de la exclusión a través del derecho a la lectura. Yuval-Davis (2014, p. 21) interroga la equivalencia analítica propuesta por Fraser (1997) entre identidades colectivas en los niveles de reconocimiento. Si bien, cada eje analítico opera de manera distinta, es también plausible recurrir a los planteamientos de Young (2005) para comprender el concepto de grupo social, política de la diferencia y emancipación social. Su contribución indaga en la redefinición de los ejes de articulación de los programas del libro y de fomento de la lectura en poblaciones significadas como colectivos vulnerables, o bien, en desventaja social, otorgando herramientas para enriquecer y potenciar la experiencia de culturización. Es menester tener presente a juicio de Young (2005) que, a pesar de que las personas puedan identificarse con ciertas categorías, cada una de ellas, tendrá una localización social diferente. Ocampo-González (2016) expone que la praxis educativa y política de la inclusión se construye en la singularidad, es decir, en la combinación de lo uno y lo múltiple. El pensamiento de multitud (Negri, 2001) es sinónimo de ilimitadas configuraciones cuyo principio diferencial es articulado a través de la afirmación productiva y diferencial como condición inmanente del sujeto. Todo ello dará paso a una forma singular que modeliza el espacio político y social de la lectura.

La constitución de un espacio político, cultural, económico, social y educativo coherente con los principios de la multitud o multiplicidad se inscribe en una ontología pluralista, como un conjunto de series singulares, deviniendo en la configuración de un acto de creación de posibilidades, un tejido de relaciones intensas y heterogéneas. A efectos de este trabajo, inscribimos la política de la multiplicidad en el contexto de educación lectora como un acontecimiento singular, esto es, un fenómeno infinitamente extenso (De Mussy \& Valderrama, 2009). La lectura concebida así, atiende a la emergencia de singularidades irreductibles en una determinada inscripción sociohistórica, cristalizando en una historicidad del presente, en un saber en constante movimiento (Bal, 2009) que propicia un conjunto de transformaciones o fracturas ante los modos dominantes y subversivos de organizar el acceso y participación a la cultura letrada y su multiplicidad de formatos de apropiación.

\section{CONCLUSIONES: POR UNA TEORÍA RADICAL DE LA LECTURA}

La lectura se convierte en una de las capacidades hermenéuticas más relevantes de la praxis ciudadana cuya fuerza de enmarcación es determinada por la capacidad de alterar el presente. En efecto, cabe destacar que esta es una de las principales debilidades que expresan las políticas de lectura y del libro, así como, los programas de animación a la lectura. A la luz de estas tensiones, se hace necesario crear nuevas prácticas lectoras concebida en términos de transformación-, que permitan recuperar la construcción de 
ciudadanía y justicia a través de la lectura y el discurso del Otro -sujeto en potencia-. De esta forma, la lectura constituye un objetivo crítico clave en la lucha por la ampliación de los campos de desarrollo democrático, se espera con ello la emergencia de un nuevo terreno que permita la cristalización de una praxis dialógica que asuma la voz de la diferencia y la multiplicidad.

En este contexto, una política de educación lectora inspirada en los fundamentos epistemológicos de la Educación Inclusiva que oriente su actividad hacia la construcción de un espacio político basado en la singularidad orientará su actividad hacia la exploración de sistemas intelectuales para atender a la diferencia sin generar procesos de diferenciación que obliguen a la exclusión de ciertos colectivos, o bien, a su supeditación. Constituye de esta forma, un espacio político basado en la diferencia, en tanto unidad relacional, que promueve el desarrollo de las personas según sus propias capacidades y no, por las nociones que definen a un determinado grupo social. Filosóficamente, inscribe sus fuerzas de actuación en un ideal de liberación concebido como eliminación de las diferencias, cristalizando así, una política emancipadora sobre educación lectora y fomento de la lectura (Ocampo-González, 2020).

Frente a estas tensiones, urge que las políticas del libro atiendan con mayor profundidad y acción estratégica, evitando contraponer marcos de valores claves en la comprensión de la diversidad, la diferencia, la inclusión, la justicia y la equidad, al tiempo que estos. De lo contrario, sus efectos serán objeto de una permeabilización imperceptible a los ejes de funcionamiento de la visión de inclusión alojada al interior del capitalismo hegemónico, esto es, mediaciones estandarizadas que poco o nada dice como mejorar la educación y crear oportunidades de culturización apropiadas a las necesidades de cada grupo social, los círculos de rendición de cuentas y culpabilidad y la inferioridad social por no alcanzar determinadas puntuaciones. En suma, estas tensiones, son reflejo de la crisis de representación -desgaste de los mapas cognitivos- que afecta a las concepciones de evaluación de la lectura.

La lectura como praxis social y política demanda la creación y movilización de nuevas racionalidades para comprender el funcionamiento de los formatos del poder, concebidas como expresiones regenerativas y performativas. Los contenidos analíticos que organizan el marco de sustentación de la teoría radical de la lectura destacan: a) investigaciones y proyecciones en el campo de la literacidad crítica y la justicia social, b) redefiniciones de la lectura y la escritura en los espacios de enseñanza-aprendizaje para la justicia educativa, la equidad, la igualdad, la inclusión y las visiones críticas requeridas para la transformación educativa, c) historia de las prácticas de enseñanza de la lectura y la escritura en el contexto de la literacidad crítica y la justicia educativa, d) dimensión política de la lectura y nuevas tendencias epistemológicas, e) estudios críticos sobre literatura y su fomento desde una perspectiva de justicia social, f) espacialidad política y ejercicio ciudadano a través de la lectura y la literacidad crítica, g) conciencia crítica y acción decolonial en el contexto de las prácticas de enseñanza de la lectura y la escritura, h) políticas de la Lectura y del Libro desde una perspectiva anti capitalista y anticolonialista, i) derechos humanos y prácticas de fomento de la lectura y j) mediación cultural para la transformación social. 
Emerge de esta forma, la necesidad de romper con la gramática escolar dominante, especialmente, con el énfasis de asimilación imperceptible con la cultura hegemónica de ciertos colectivos concebidos como no culturalizados o no competentes en materia de lectura. ¿Qué elementos permiten edificar una arquitectura educativa, social y cultural cuyo centro de articulación emerja desde una conciencia ontológica de naturaleza pluralista? Un formato escolar y cultural con estas características contrapone, incluso, las propuestas más esperanzadoras retóricamente, ratificadas a través las políticas de diversos ministerios de Educación en Latinoamérica y Europa.

Una política de educación lectora comprometida con cada uno de estos objetivos analíticos y políticos evitará "restarle importancia a la relación históricamente elaborada entre lenguaje y poder, los educadores críticos no han logrado un discurso que articule cuestiones de identidad, lugar, pedagogía e historia con un lenguaje de visión y vida pública" (McLaren y Giroux, 1998, p. 18). En una línea postestructuralista se interesa por comprender cómo educar a las múltiples subjetividades a través de la lectura y “cómo el lenguaje se utiliza tanto para legitimar como para marginar diferentes posturas sobre un tema o cómo el conocimiento no sólo mistifica sino funciona para generar identidades, deseos y necesidades" (McLaren, 2004, p. 25), cristalizando en prácticas lectoras de la multitud.

Por último, en futuros trabajos y propuestas se indagará en la siguiente idea: los colectivos vulnerables o vulnerabilizados no es que no disfruten de la lectura, lo hacen en un formato diferente basado en medios de apropiación y tecnologías que desafían y ponen en tensión los marcos de valores propios de la cultura letrada occidental hegemónica. Su producción del deseo es lo que hace que la gramática escolar dominante los devalúe y subalternice. La producción del deseo se convierte así, en un ámbito analítico clave en la articulación de una agenda oposicional en materia de educación lectora. Una aproximación a las cartografías lectoras del presente abordará la producción del deseo como elemento de determinación de los intereses de la multiplicidad de lectores a los que se dirige.

\section{REFERENCIAS BIBLIOGRÁFICAS}

Anthias, F. \& Yuval-Davis, N. (1983). Contextualizing feminism: gender, ethnic and class divisions, Feminist Review, 15, 62-75.

Araújo, A. M., \& Cardozo, A. (2016). Tiempos acelerados y espacios nómades de la hipermodernidad: Reflexiones abiertas. Psicología, Conocimiento y Sociedad, 6 (2), 209-222.

Bal, M. (2009). Conceptos viajeros en las humanidades. Una guía de viaje. Murcia: Cendeac.

Ball, S. J., \& Míguez, N. (1989). La micropolítica de la escuela: hacia una teoría de la organización escolar. Barcelona: Paidós.

Bauman, Z. (2007). Los retos de la educación en la modernidad líquida. Barcelona: Gedisa.

Beck, U. (1998). La sociedad del riesgo: hacia una nueva modernidad. Barcelona: Paidós ibérica. 
Bombini, G. (2008). La lectura como política educativa. Revista iberoamericana de educación, 46 (1), 19-36.

Brah, A. (2014). Pensando en y a través de la Interseccionalidad. En Zapata, M., García, S., Chan de Avila, J. (Edit.). La interseccionalidad en debate. Actas del Congreso Internacional "Indicadores interseciconales y Medidas de Inclusión Social en Instituciones de Educación Superior”, 14-20. Berlín: Aimee Heredia.

Casanova, G. S., \& Navas, J. J. L. (2015). Política global más allá de lo nacional. Reforma educativa (LOMCE) y el régimen de estandarización (OECD). Bordón. Revista de pedagogía, 67 (1), 135-148.

Chartier, A. M. (2004). Enseñar a leer y escribir: una aproximación histórica. México: Fondo de Cultura Económica.

De Mussy, L. \& Valderrama, M. (2009). Historiografía postmoderna: conceptos, figuras y manifiestos. Santiago: RIL/Universidad Finnis Terrae.

Edmondson, J., \& Shannon, P. (2002). Politics of reading: The will of the people. The Reading Teacher, 55 (5), 452-454.

Fernández Liria, C., García Fernández, O., \& Galindo Ferrández, E. (2017). Escuela o barbarie: entre el neoliberalismo salvaje y el delirio de la izquierda. Madrid: AKAL.

Fernández-Cano, A. (2016). Una crítica metodológica a las evaluaciones PISA. RELIEVE-Revista Electrónica de Investigación y Evaluación Educativa, 22 (1).

Fernández-González, N. (2015). PISA como instrumento de legitimación de la reforma de la LOMCE. Bordón. Revista de pedagogía, 67 (1), 165-178.

Fraatz, J. M. B. (1987). The politics of reading: Power, opportunity, and prospects for change in America's public schools. Amsterdam y Nueva York: Teachers College Press.

Fraser, N. (1997). Justice Interruptus. Nueva York: Routledge

Garcés, M. (2017). Nueva ilustración radical. Barcelona: Anagrama.

Giroux, H. A. (1992). Teoría y resistencia en educación: una pedagogía para la oposición. Buenos Aires: Siglo XXI.

Han, B. C. (2017). La sociedad del cansancio. Barcelona: Herder Editorial.

Hill Collins, P. (1990). Black feminist thought: knowledge, consciousness, and the politics of empowerment. Londres: Routledge.

Hirtt, N. (2010). La educación en la era de las competencias. Revista electrónica interuniversitaria de formación del profesorado, 13 (2), 108-114.

hooks, B. (2017). El feminismo es para todo el mundo. Madrid: Traficante de Sueños.

Kaplan, C. (2012). Buenos y malos alumnos. Descripciones que predicen. Buenos Aires: Aique.

Lazzarato, M. (2006). Por una política menor. Acontecimiento y política en las sociedades de control. Madrid: Traficante de Sueños.

Lorey, I. (2008). Gubernamentalidad y precarización de sí. Sobre la normalización de los productores y las productoras culturales. En Producción cultural y prácticas instituyentes. Lineas de ruptura en la crítica institucional, 57-78. Madrid: Traficantes de Sueños.

McLaren, P. (2004). La vida en las escuelas. Una introducción a la pedagogía crítica en los fundamentos de la educación. Buenos Aires: Siglo XXI.

McLaren, P. \& Giroux, H. (1998). Sociedad, cultura y educación. Madrid: Editorial Miñó y Dávila Editores-Instituto Paulo Freire.

Negri, A. (2001). Marx más allá de Marx. Cuaderno de trabajo sobre los Grundrisse. Madrid: Akal. 
Ocampo-González, A. (2020). Prácticas letradas en clave interseccional y post-colonial: por el derecho a la diferencia y a la reivindicación de la ciudadanía cultural. Santiago de Chile: Ediciones CELEI.

Ocampo-González, A. (2016). Interseccionalidad y derecho a la lectura. Aportes teóricometodológicos para el fortalecimiento de la ciudadanía y el fomento de la lectura desde una perspectiva de Educación Inclusiva. Santiago de Chile: Ediciones CELEI.

Ocampo-González. A. \& López-Andrada, C. (2020). Transformando los espacios de lectura. Una perspectiva crítica e inclusiva de las prácticas lectoras contemporáneas. Educare, 24 (1), 192-212.

OECD (2009). Assessment Framework: Key Competencies in Reading, Mathematics and Science. Paris: OECD Publishing.

Perfetti, C. A. (1991). The psychology, pedagogy, and politics of reading. Psychological Science, 2 (2), 70-83.

PIAAC (2013). Programa Internacional para la Evaluación de las Competencias de la población $\quad$ adulta. Vol. http://www.mecd.gob.es/dctm/inee/internacional/piaac/piaac2012.pdf?document $\mathrm{Id}=0901 \mathrm{e} 72 \mathrm{~b} 8181 \mathrm{~d} 500$

PISA (2009). Programa para la evaluación internacional de los alumnos. http://www.mecd.gob.es/dctm/ievaluacion/internacional/informe-espanol-pisaera-2009.3.pdf?documentId $=0901 \mathrm{e} 72 \mathrm{~b} 80 \mathrm{~d} 5 \mathrm{a} 81 \mathrm{e}$

Prais, S. J. (2003). Cautions on OECD's recent educational survey (PISA). Oxford Review of Education, 29 (2), 139-163.

Rizvi, F., \& Lingard, B. (2009a). Globalizing education policy. Londres: Routledge.

Rizvi, F., \& Lingard, B. (2009b). The OECD and global shifts in education policy. En Cowen R., Kazamias A.M. (eds), International handbook of comparative education, 437-453. Springer, Dordrecht.

Sousa, B. (2010). Descolonizar el saber, reinventar el poder. Montevideo: Ediciones Trilce, Extensión Universitaria de la República.

Spivak, G. (2008). Estudios de la Subalternidad. Deconstruyendo la historiografía. En Mezzadra,S., Spivak, G., Tapadle, Ch., Sota, E., Hall, S. Chakrabarty, D., Mbembe, A., Young, R., Puwar, N. \& Róala, F. (eds.), Estudios Postcoloniales. Ensayos fundamentales, 33-68. Madrid: Traficante de Sueños.

Taylor, S., Henry, M., Lingard, B., \& Rizvi, F. (1997). Educational policy and the politics of change. Londres y Nueva York: Routledge.

Turner, R. (2006). El Programa Internacional para la Evaluación de los Alumnos (PISA). Una perspectiva general. Revista de Educación, número extraordinario, 45-74.

Young, I.M. (2002). Justicia y Política de la Diferencia. Valencia: Cátedra.

Young, I.M. (2005). On Female Body Experience, "Throwing Like a Girl" and Other Essays. Oxford: Oxford University Press.

Yuval-Davids, N. (2014). Más allá de la dicotomía del reconocimiento y la redistribución: interseccionalidad y estratificación. En Zapata, M., García, S., Chan de Avila (coords.), La interseccionalidad en debate, 21-34. Berlín: MISEAL. 